\title{
Abstracts from the 6th Annual Healthcare Transition Research Consortium Conference, Houston, TX, USA, 2014
}

\author{
Introduction \\ Parag Shah \\ E-mail: pshah@luriechildrens.org
}

There are many groups conducting work on transition throughout the country. The field lends itself to innovative, multidisciplinary approaches to cover a complex topic. We would like to present some samples of work being done around the country relating to transition for populations that may be of interest to the readers of the journal. The abstracts are selected from The 6th Annual Healthcare Transition Research Consortium Conference, Houston 2014. The Consortium represents an international group of multidisciplinary Researchers in Healthcare Transitions, including physicians, nurses, social workers, therapists, and allied health professionals. The abstracts for the conference were initially selected through a blinded peer review process by members of the administrative committee of the consortium. From the abstracts selected for the conference at large, a few were selected by a subgroup of reviewers based on significance for this journal.

\section{Community Participation of Youth and Young Adults with Special Health Care Needs (YYASHCN)}

\section{Mary R. Ciccarelli \\ E-mail: mciccare@iu.edu}

Background: The International Classification of Functioning, Disability, and Health (ICF) describes body function, a person's capacity to do activities, and actual participation in his/her usual environment. Health conditions and personal and environmental factors affect the level of participation. One goal for YYASHCN in their transition to adult life is to maintain or achieve inclusive participation in the community. Therefore, an understanding of function, activity and participation is important for those designing transition services for YYASHCN.

Methods: A statewide non-categorical transition support program mailed intake surveys to new families prior to their first visit for transition consultation and support. Surveys were developed to assess current community participation and impacting personal and environmental factors. Questions regarding needs and limits were adapted from the National Health Information Survey. Health status and life satisfaction questions are similar to those used in the Child Health Questionnaire. Three hundred twenty-three consented parent/caregiver surveys were returned via mail or at the first appointment between April 2010 and April 2014.

Results: Ninety-one percent of parents reported their youth needed assistance, such as with personal care needs $(58 \%)$, routine needs $(84 \%)$, and adaptive equipment $(53 \%)$. Two-thirds $(68.8 \%)$ of respondents stated their youth participated in social, religious or recreational activities outside of the home, despite $76 \%$ of these participants having impairments that were limiting (physical, mental or emotional condition). Parents reported that $47.3 \%$ of youth watched 3 or more hours of television daily. High and low volume television viewers participated in community activities at the same rate (70 vs $68 \%$ ). Environmental factors, including building design, lighting, sound, equipment, sidewalks or transportation, were noted as limiting in three-quarters of youth (76.7\%). Personal factors, such as lack of self-confidence or feeling unsafe, were noted as limiting (64.7\%). Eighty-one percent of youth who participate in community activities report life satisfac- 
tion, compared with $65 \%$ of those who do not participate.

Conclusion(s): The participants in the state transition support program have high needs for care and assistance. One-third of surveyed YYASHCN do not participate in community activities beyond school attendance. Environmental and personal factors both contribute to limiting participation. Life satisfaction is associated with community participation. Transition programs, in addressing the importance of community participation for its potential impact on life satisfaction, should attend to individual barriers.

Social Support for Adolescents with Chronic Conditions and its Association to Patient-Reported Outcomes

Gary Maslow

E-mail: gary.maslow@duke.edu

Purpose: The purpose of this study was to examine the relationship between social support from parents or friends with patient reported outcomes (health-related quality of life (QoL) and self-esteem) for adolescents with a wide variety of childhood-onset chronic health conditions.

Methods: A web-based survey was conducted among 13-16 years old adolescents with a wide variety of chronic health conditions, before arriving at a pediatric camp. Measures included a pediatric quality of life scale (PedsQL ${ }^{\mathrm{TM}}$ ) and the Rosenberg Self-esteem scale. Also, participants completed a survey about the social support they receive from parents and friends. Support was divided into instrumental (concrete action) vs. emotional support and into directive (my friends tell me what to do) vs. nondirective (my friends let me decide what to do) support. The association between different forms of social support and QoL and self-esteem were examined using multivariate regression.

Results: We enrolled 135 adolescents (55\% response rate) with 16 distinct health conditions. Nondirective social support from friends was associated with higher quality of life and self-esteem. Nondirective emotional support from parents was associated with higher quality of life, but not related to self-esteem.

Conclusions: Greater social support from friends and parents, particularly nondirective emotional support, is correlated with higher self-esteem and quality of life for adolescents with chronic conditions.
Preliminary Findings: Descriptive Analysis of HCT Services Provided to Adolescents and Emerging Adults with Spina Bifida

Cecily L. Betz, Kathryn Smith, Alex Van Speybroeck, Natalie E. Rivera, Saba Saghafi, Jeannie Lee

E-mail:cbetz@chla.usc.edu

Background: Acknowledgement of the health care transition (HCT) service needs of adolescents and emerging adults with special health care needs (AEASHCN) has generated considerably more attention during the past two decades. Widespread international efforts are underway to implement and test the effectiveness of HCT service models. The science and practice of HCT are in the seminal stages of development. For example, findings of a systematic review of the research of AEA-SHCN attitudes toward HCT to better understand their service needs revealed limited evidence. The preponderance of studies were conducted in international settings wherein AEA-SHCN have universal access to health care, unlike the United States. In contrast, relatively few studies were conducted in the United States. Furthermore, the external validity of these studies is limited due to small sample sizes of AEA-SHCN who represent few diagnostic conditions (author, blinded). Evidence is needed to better understand the phenomenon of HCT. Empirical data are needed to gain understanding of the biopsychosocial factors that support or inhibit AEA-SHCN transition to adulthood and the transfer of care to adult services. Many gaps exist in the body of HCT science that are essential to the development of evidence-based HCT practice. Examples of the issues that challenge the development of empirically supported HCT models are the lack of psychometrically valid and reliable instruments, problems with measuring HCT outcomes, and the lack of well defined HCT service models that can be replicated.

Purpose: To that end, descriptive data are needed to better understand the characteristics and profile of services that constitute HCT interventions. The model of HCT services described here is based upon a nursing practice approach that incorporates an interdisciplinary framework of services.

Findings: This poster presents an overview of this nurse-led interdisciplinary model of care that was implemented in January 2011. Findings presented in this poster are based upon a 150 youth with spina bifida, ages 10 to 20 years of age who have been enrolled into the program. Describe data reported will include a 
summary profile of the demographics and clinical characteristics of the youth enrolled in the SB HCT Program.

Conclusion: A descriptive profile will be reported as to the type of problems/interventions identified using the Health Care Transition Research Consortium Health Care Transition Model and nursing interventions using the Omaha System descriptors.

\section{Improving Transition to Adulthood from a Pedi- atric Complex Care Service \\ Kitty O'Hare \\ E-mail: Frances.OHare@childrens.harvard.edu}

Background: Youth with complex medical needs experience many barriers as they graduate from pediatric to adult care. Pediatricians report difficulty in identifying subspecialists and primary care physicians for their complex patients. Further, families struggle with the advanced care coordination tasks required during the young adult period.

Program Development: In 2013, Boston Children's Hospital implemented a new transition support service called BRiDGEs (Building Relationships and Developing Goals with Emerging Adults). The BRiDGEs service is staffed by med-peds physicians with expertise in transitioning youth to adult care. BRiDGEs is partnering with the hospital's existing Complex Care Service (CCS) to improve its care of young adults. CCS includes an inpatient ward service as well as a large ambulatory program comprised of multiple subspecialty clinics. The BRiDGEs service provides inpatient consults to patients ages 18-35 years who are hospitalized on the CCS ward. Consults may address adult-type medical issues, such as concern for DVT, or they may address transfer issues, such as identifying options for an adult primary care physician. In the ambulatory clinics, a BRiDGEs physician provides consultation visits that address self-management skills, family coping skills, options for transfer to adult care, and care coordination.

Program Evaluation: Informal interviews with providers who have utilized the BRiDGEs program have been quite positive. A particular strength of the program is the ability of med-peds providers to connect patients with adult primary care physicians and adult subspecialists. A series of seminars are planned to improve the comfort of CCS providers in providing general young adult care as well as transition care.

Conclusions: There is a need to improve the quality of adult transitions from pediatric complex care programs. The BRiDGEs/CCS collaboration is one promising model for supporting the needs of youth with

\author{
Divergent Factors Related to Quality of Life in \\ Family Members of Young Adults with Spina Bifida \\ Kathleen Sawin \\ E-mail: sawin@uwm.edu
}

Background: Understanding factors related to quality of life (QoL) for family members is fundamental to designing interventions to optimize outcomes. The purpose of this study is to determine differing factors related to QoL in families who have a young adult (YA) with spina bifida. The Ecological Model of Adaptation in Spina Bifida was the framework for the study.

Methods: In this descriptive correlational study, 30 families prticipating in a Spina Bifida Transition Program (SBTP) for young adults in a large Midwestern city, completed surveys before the first SBTP appointment. Participants were YAs ages 18-35 (M = 22 ; sd $=4$ ) and their parents. The YAs were primarily students or unemployed, $45 \%$ attended an educational program in the last 6 months, two had been married, $51 \%$ had 4 or more friends and $63 \%$ see their friends 5 times a month or more. Parents were primarily mothers and Caucasian. QoL was measured with two questions addressing participants' perceived overall individual and family QoL. Measures of other concepts had established reliability and validity. Pearson correlations were used to assess relationships.

Results: For each participant individual and family QoL scores were strongly correlated but the relationship between YA and their parents QoL scores was small. YA factors related to QoL included attitude, communication and problem-solving self-efficacy, family satisfaction, perceived health, and perceived health competence ( $r=0.41$ to 0.73 ). In contrast, parent factors related to QoL items included perceived health of the YA, YA functional status, and YA selfmanagement ( $r=0.41-0.57)$.

Conclusions: Perceived health of the YA was the only common factor related to the QoL outcomes for YAs and their parents. Other factors explaining QoL in YAs and their parents differed dramatically. For YAs, resilience factors (attitude, self-efficacy, family satisfaction, perceived health competence) were important, while for parents, their YA's functional status and independence in self-management were significant associations. 
Needs Identified by Young Adults with Spina Bifida Participating in a Transition Program

Kathleen Sawin

E-mail:sawin@uwm.edu

Background: Pediatric and adult providers partnered to create a Spina Bifida Transition Program in a tertiary care setting. The purpose of this analysis was to determine the reliability of an instrument to measure participants' needs and to evaluate the relationships of needs to self-management.

Methods: Survey data was collected from 30 participants without intellectual disabilities before the transfer to adult health care. Instruments included the Kennedy SB Needs Questionnaire (SBNQ), the Canadian Occupational Performance Measure (COPM) and the Adolescent/Young Adult Self-Management Scale (AMIS II). The 67-item SBNQ, (scale of 1-7 with higher scores $=$ larger needs) has not been used with young adults in transition to date.

Results: Mean participant age was 22 years (range 1835). Transition patient were primarily Caucasian and $53 \%$ were male. Seven of the SBNQ's 8 subscales had acceptable reliabilities ( $\alpha=0.71$ to 0.93 with 5 of the 7 subscales above $\alpha=0.84)$. The mean subscale scores ranged from a low need for counseling (mean $=3.4$, $\mathrm{SD}=1.5)$ to a higher need for policy reform (mean $=$ $4.3, \mathrm{SD}=1.5$ ) with 5 of the 7 subscales in the 4.0 to 4.3 range. Lower needs in the areas of counseling, socialization and independence were moderately related to higher AMIS II scores ( $r=0.35$ to 0.48 ). Participants identified a mean of 3.8 COPM goals.

Discussion: The SBNQ was reliable and captured individual variation in need. However, the COPM was more effective at identifying priority needs/goals. The finding that decreased support needs and increased socialization/independence were related to higher selfmanagement behaviors suggest area for future interventions.

Conclusion: Determining the needs of transition age young adults and their families is critical. The COPM, although more time consuming, addressed performance, importance and satisfaction in individualized goals and may be more useful in clinical practice.

Youth with Cerebral Palsy: Successes and Challenges of Establishing a Sub-Specialty Transition Clinic

Michelle Romano-Shami

E-mail: Michelle.Romano@NYUMC.ORG
Purpose: Transition/transfer of Youth with Special Health Care Needs is an important issue. Few programs offer transition/transfer from pediatric to adult sub-specialists. We faced many successes and challenges in establishing a Transition to Adulthood Clinic (TAC) for patient transfer from pediatric to adult subspecialists and focused on patients with cerebral palsy (CP).

Methods: Multidisciplinary staff met biweekly to discuss patient care needs, a model for TAC, logistics/planning, addressing barriers, financial sustainability, and outcome measures. A list of active patients with CP was categorized to include the following age groups: 18 years and above (adult), and under 18 years of age. Inclusion criterion was at least one ambulatory visit in the Center for Children from September 2011 through December 2013.

Results: Of the 1,888 total patients, 671 patients (36\%) were adults and 298 patients $(16 \%)$ were $14-17$ years of age. Successes included: creation of an interdisciplinary team, adaptation of a pre-established model from another institution, development of relationships with adult providers, development of a tool to identify sub-specialty consults based on diagnostic categories, and development of tools to measure patient progress/outcomes. Challenges included financial sustainability, willingness of adult providers to treat this population, patients/families to transfer care, and pediatric providers to relinquish care.

Conclusion: According to the 2009-2010 National Survey of Children with Special Health Care Needs, only $40 \%$ of respondents reported that they have discussed transition issues with their physician and, far less, $16 \%$ have discussed and developed a plan for addressing transition/transfer to adult providers. As 52\% of our patients with $\mathrm{CP}$ fell in the age range of 14 years and above, the need for a transition program was imperative.

Significance: Establishing a program to facilitate the transition/transfer of patients with $\mathrm{CP}$ from pediatric to adult sub-specialists is critical to addressing the current and future needs of this population.

\section{Framework for Self-Management Education Erin Holbrook \\ E-mail: Erin.Holbrook@cchmc.org}

Background: In order to assess an initial level of selfmanagement skills in our inflammatory bowel disease (IBD) population, the IBD Transition of Care team at Cincinnati Children's Hospital Medical Center be- 
gan systematically using the Transition Readiness Assessment Questionnaire (TRAQ) with patients aged 16 years and above. Based on TRAQ data gathered from 66 patients over a five month period in 2013, we found the average patient has mastered only 9 out of 20 TRAQ skills. Throughout the process of establishing a greater focus on goal setting, tracking patients' progress toward goals and timely reassessments for mastery of self-management skill; we determined it is crucial to focus on skills vital to successful transition as indicated by patients, parents and clinicians.

Methods: In 2014, 20 patients, 20 parents and 20 clinicians in our IBD Center were polled to identify which five TRAQ skills were deemed to be essential for successful transition. Using a multi-voting system, the top five skills were chosen from this aggregate data. Results: Of all transition readiness skills listed, being able to communicate with one's physician about unusual changes in health was most often rated by respondents as being important for successful transition $(68.3 \%)$. Following up on referrals (e.g., labs, checkups) ranked second (63.3\%) whereas skills associated with medication management (e.g., taking medications correctly, reordering medications) ranked 3rd and 4th (61.6\% and $60 \%$ respectively). The 5th skill considered most important was scheduling medical appointments $(53.3 \%)$.

Conclusion: Targeting the skills deemed to be paramount by patients, parents and clinicians for successful transition; we are building an educational framework utilizing content from the IBD Self-Management Handbook and the NASPGHAN Transition Checklist. This will better position our team to help patients master those skills necessary for an effective transition. 